\title{
Multi-dimensional Mutual Information Based Robust Image Registration Using Maximum Distance-Gradient-Magnitude
}

\author{
Rui Gan and Albert C.S. Chung \\ Lo Kwee-Seong Medical Image Analysis Laboratory, \\ Department of Computer Science, \\ The Hong Kong University of Science and Technology, Hong Kong \\ \{raygan, achung\}@cs.ust.hk
}

\begin{abstract}
In this paper, a novel spatial feature, namely maximum distance-gradient-magnitude (MDGM), is defined for registration tasks. For each voxel in an image, the MDGM feature encodes spatial information at a global level, including both edges and distances. We integrate the MDGM feature with intensity into a two-element attribute vector and adopt multi-dimensional mutual information as a similarity measure on the vector space. A multi-resolution registration method is then proposed for aligning multi-modal images. Experimental results show that, as compared with the conventional mutual information (MI)-based method, the proposed method has longer capture ranges at different image resolutions. This leads to more robust registrations. Around 1200 randomized registration experiments on clinical 3D MR-T1, MR-T2 and CT datasets demonstrate that the new method consistently gives higher success rates than the traditional MI-based method. Moreover, it is shown that the registration accuracy of our method obtains sub-voxel level and is acceptably high.
\end{abstract}

\section{Introduction}

A key issue in the medical imaging field is multi-modal image registration, which can integrate complementary image information from different modalities. The task of image registration is to reliably identify a geometric transformation to accurately align two images.

A crucial element in the registration process is a similarity measure to determine how well the images match with each other through a hypothesized spatial transformation. General promising results have shown that mutual information (MI) as a voxel intensity-based similarity measure is well-suited for multi-modal image registration [1,2]. However, it has been suggested that the conventional MI-based registration can result in misalignment for some cases [3, 4, and then room for improvement exists. The standard MI measure only takes intensity values into account. Therefore, a known disadvantage is the lack of concern on any spatial information (neither local nor global) which may be present in individual 
images to be registered [5, 6]. As a simple illustration, a random perturbation of image points identically on both images results in unchanged MI value as that of the original images.

Several researchers have proposed adaptations of the MI-based registration framework to incorporate spatial information of individual images. Butz et al. 7] applied MI to edge measure (e.g., gradient magnitude) space, which was meant to align object surfaces in images. However, MI based on edge measure is sensitive to the sparseness of joint edge feature histograms. This may increase the difficulty of the optimization procedure. Pluim et al. 4 incorporated spatial information by multiplying the conventional MI measure with an external local gradient term to ensure the alignment of locations of tissue transitions in images. The probing results indicated that the registration function of the combined measure was smoother than that of the standard MI measure. But this approach does not actually extend the MI based similarity measure. Moreover, Rueckert et al. 6] exploited higher-order mutual information for 4D joint histograms. To include local spatial information present by neighboring point pairs, the $4 \mathrm{D}$ joint histograms were built on the co-occurrence of intensity pairs of adjacent points. This method was shown to be robust with respect to local intensity variation. However, only one neighbor is considered at a time in this approach and plenty of spatial information which may be present globally or within large neighborhood system has been ignored.

In this paper, a new spatial feature, namely maximum distance-gradientmagnitude (MDGM), is defined for registration tasks. The MDGM feature encodes spatial information for each voxel in an image at a global level, which is about the distance of a voxel to a certain object boundary. In order to improve the conventional MI-based registration framework, we integrate the MDGM feature with intensity to form a two-element attribute vector for each voxel in individual images. Then, multi-dimensional mutual information is exploited as a similarity measure on the attribute vector space. To increase computation efficiency and robustness of the proposed method, the registration procedure is a multi-resolution iterative process.

Based on the results on clinical 3D MR-T1, MR-T2 and CT image volumes, it is experimentally shown that the proposed method has relatively longer capture ranges 1 than the conventional MI-based method at different image resolutions. This can obviously make the multi-resolution image registration more robust. Moreover, the results of around 1200 randomized registration experiments reveal that our method consistently gives higher success registration rates than the traditional MI-based method. Finally, it is demonstrated that our method can obtain acceptably high registration accuracy in sub-voxel level.

The organization of the paper is as follows. Section 2 formulates spatial information as a novel MDGM feature. Our multi-modal image registration method is proposed in Section 3. Some implementation details are given in Section 4.

\footnotetext{
${ }^{1}$ Capture range represents the range of alignments from which a registration algorithm can converge to the correct maximum.
} 
Section 5 presents the experimental results and discussions. The conclusion is drawn in Section 6 .

\section{Spatial Feature Definition}

Given an image pair and a geometric transformation, we aim at evaluating a novel multi-dimensional mutual information based registration criterion. In our proposed approach, each voxel in the image has a two-element attribute vector. The first element is the conventional voxel intensity, while the second one is a newly designed spatial feature term, namely maximum distance-gradientmagnitude (MDGM), for incorporating spatial information at a global level within individual images. Compared with the traditional local gradient magnitude feature, the MDGM feature can encode local edge information, as well as globally defined spatial information about the distance of a voxel to a certain object boundary. Although it can be similar to the distance transform [8, the distance transform is normally applied to binary images, while the proposed MDGM feature directly processes original intensity images and does not rely on segmentation. Moreover, unlike the sparseness of gradient magnitude feature, the MDGM feature varies smoothly and gradually from object boundaries towards homogeneous image regions.

\subsection{Maximum Distance-Gradient-Magnitude (MDGM)}

Gradient magnitude represents spatial information in an image. However, the traditional gradient magnitude operator is locally defined and normally used to detect the amplitude object boundaries where voxels change their gray-level suddenly. By deriving gradient magnitude map, voxels at object boundaries, which may only occupy a very small proportion of the whole image volume, would give large values. On the other hand, a large amount of voxels (i.e. voxels within background regions and anatomical structures) would give small and almost constant values. Consequently, such gradient magnitude feature of an image can be sparse and insufficient for voxel-based image registration [9].

In this section, we define a new spatial feature, maximum distance-gradientmagnitude (MDGM). It contains not only local edge information, but also spatial information at a global level, which is about the distance of a voxel to a certain object boundary. Moreover, the MDGM feature varies smoothly and gradually from object boundaries towards homogeneous image regions.

We begin by defining a distance-gradient operator, $\nabla_{d}$, on two voxels in an image. Given an image $I(\mathbf{v})$, where $\mathbf{v}=(x, y, z)$ denotes voxel position, the distance-gradient of two different voxels, $\mathbf{v}_{1}$ and $\mathbf{v}_{2}$, is defined as

$$
\nabla_{d} I\left(\mathbf{v}_{1}, \mathbf{v}_{2}\right)=\left(I\left(\mathbf{v}_{1}\right)-I\left(\mathbf{v}_{2}\right)\right) \frac{\mathbf{v}_{1}-\mathbf{v}_{2}}{\left|\mathbf{v}_{1}-\mathbf{v}_{2}\right|^{2}}
$$

Then, a $M D G M$ map, $G(\mathbf{v})$, of the image can be derived by using

$$
G(\mathbf{v})=\max _{\mathbf{v}^{\prime} \in \Omega}\left|\nabla_{d} I\left(\mathbf{v}^{\prime}, \mathbf{v}\right)\right|,
$$




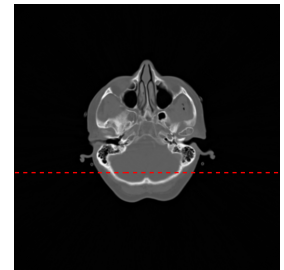

(a) A CT slice

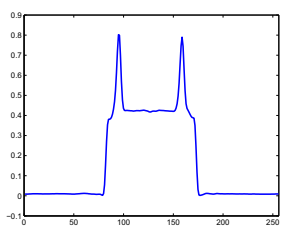

(e)

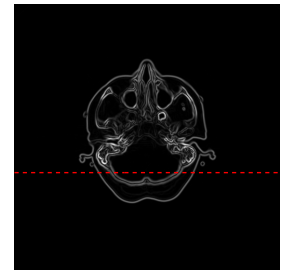

(b) GM map

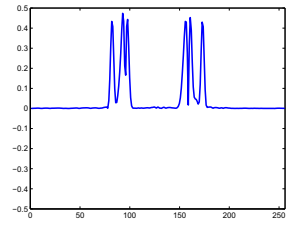

(f)

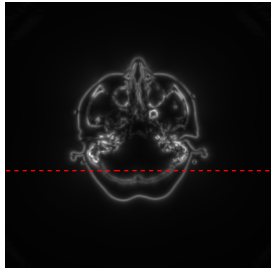

(c) MDGM map

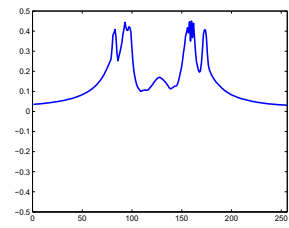

(g)

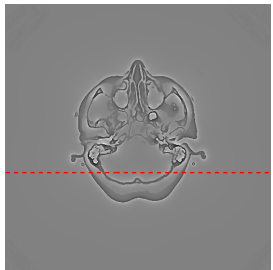

(d) Signed-MDGM map

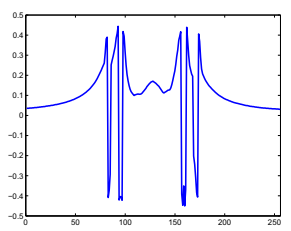

(h)

Fig. 1. (a) - (d) are slices respectively selected from a clinical CT image volume and its corresponding GM, MDGM and signed-MDGM maps. (e) - (g) are value profiles of lines in (a) - (d), which are marked as dashed lines

where $\Omega$ is the image domain. Following this formulation, when a voxel is at or very close to object boundary, its MDGM value would be large and can approximate the traditional local gradient magnitude. On the other hand, when voxel position varies from boundaries towards interiors of homogenous regions (either background regions or anatomical structures), the MDGM value smoothly and gradually decreases. With this property, the MDGM map of homogenous regions can provide global and detailed spatial information (which is about the distance of a voxel to a certain object boundary), and therefore is superior to the local gradient magnitude map.

As a comparative illustration, we individually computed the traditional local gradient magnitude (GM) and MDGM maps of a clinical CT image volume obtained from the Retrospective Registration Evaluation Project (RREP) 2 . A slice from the volume is shown in Figure 1a, while Figures 1 $\mathrm{b}$ and 10 respectively present the corresponding slices from the GM and MDGM maps. (Note that values from individual images are re-scaled to $[0,1]$ for a fair comparison.) It is observed that the GM map can only exhibit sharp edge information. In addition, much more structural information can also be found in the MDGM map. For instance, regions close to boundaries in Figure 1 suggest much more information than those in Figure 1b. However, due to the limitation of image quality, smooth changes within the background regions and anatomical structures may not be clearly displayed in Figure 1:.

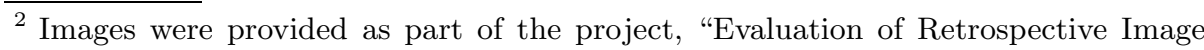
Registration", National Institutes of Health, Project Number 1 R01 NS33926-01, Principle Investigator, J. Michael Fitzpatrick, Vanderbilt University, Nashville, TN. 
For a detailed description, Figures 10 - 18 respectively present the value profiles of the same line (marked as dashed lines) in Figures 1a - 1k. As suggested by the figures, feature values in Figure 1f are very sparse, where the overwhelming majority are small and constant. Contrarily, for Figure $1 \mathrm{~g}$, the value variation from boundaries towards homogenous regions is smooth and gradual. It is worth noting that, although there is little intensity variation at the middle of the line in Figure 11, an evident and smooth saddle can be found in Figure 1p located at the corresponding position. The raised white boundary slightly below the line cause this saddle. It is because, unlike the local gradient magnitude operator, the MDGM operator is globally defined.

\subsection{Signed-MDGM}

In order to make the MDGM map be capable of distinguishing voxels of objects with different intensities, we further introduce the signed-MDGM map, $\hat{G}(\mathbf{v})$, as follows,

$$
\hat{G}(\mathbf{v})=\operatorname{sign}(I(\hat{\mathbf{v}})-I(\mathbf{v})) \cdot\left|\nabla_{d} I(\hat{\mathbf{v}}, \mathbf{v})\right|,
$$

where $\hat{\mathbf{v}}=\arg \max _{\mathbf{v}^{\prime}}\left|\nabla_{d} I\left(\mathbf{v}^{\prime}, \mathbf{v}\right)\right|$, and the function $\operatorname{sign}(\cdot)$ indicates the sign of the difference. According to this modified definition, a voxel of relatively low intensity would have a positive MDGM value and vice versa. (It should be noticed that, for a fixed $\mathbf{v},|\hat{G}(\mathbf{v})|=G(\mathbf{v})$.)

As a comparison, Figures $1 \mathrm{~d}$ and $1 \mathrm{~h}$ respectively show the corresponding slice and value profile from the signed-MDGM map of the aforementioned CT image volume. Obviously, the signed-MDGM map presents all the properties shown in the MDGM map. Furthermore, as shown in Figure 1h, voxels of objects with different intensities are distinguishable. Hereafter, we adopt the signed-MDGM feature to represent spatial information for registration tasks.

\section{Multi-modal Image Registration}

As we have discussed above, the signed-MDGM feature encodes spatial information at a global level. We associate it with voxel intensity to form a two-element attribute vector for registration. Given two images, in order to measure the degree of dependence of the attribute vector space, multi-dimensional (i.e. 4D) mutual information (MI) is exploited as a similarity measure.

\subsection{Multi-dimensional Mutual Information}

Suppose that $I^{r}$ and $I^{f}$ are the intensity domains for the reference and floating images respectively, and $\hat{G}^{r}$ and $\hat{G}^{f}$ are their signed-MDGM domains. Given a rigid transformation $\mathbf{T}$, the $4 \mathrm{D}$ joint histogram $h_{\mathbf{T}}\left(I^{f}, \hat{G}^{f}, I^{r}, \hat{G}^{r}\right)$ over the sampling set $\mathcal{V} 3$ can be approximated by either Parzen windowing or histogramming [10]. Histogramming is employed in this paper because the approach is

\footnotetext{
${ }^{3}$ The sampling set $\mathcal{V}$ can be all voxels in the floating image or a subset.
} 
computationally efficient. That is, $h_{\mathbf{T}}\left(I^{f}, \hat{G}^{f}, I^{r}, \hat{G}^{r}\right)$ is constructed by binning the attribute vector pairs $\left(I^{f}(\mathbf{v}), \hat{G}^{f}(\mathbf{v}), I^{r}(\mathbf{T} \cdot \mathbf{v}), \hat{G}^{r}(\mathbf{T} \cdot \mathbf{v})\right)$ for all $\mathbf{v} \in \mathcal{V}$. The trilinear partial volume distribution interpolation [1] is exploited to update the joint histogram for non-grid alignment.

Then the 4D mutual information registration criterion is evaluated by using

$$
\operatorname{MI}(\mathbf{T})=\sum_{I^{f}, \hat{G}^{f}, I^{r}, \hat{G}^{r}} p_{\mathbf{T}}\left(I^{f}, \hat{G}^{f}, I^{r}, \hat{G}^{r}\right) \log _{2} \frac{p_{\mathbf{T}}\left(I^{f}, \hat{G}^{f}, I^{r}, \hat{G}^{r}\right)}{p_{\mathbf{T}}\left(I^{f}, \hat{G}^{f}\right) \cdot p_{\mathbf{T}}\left(I^{r}, \hat{G}^{r}\right)},
$$

where

$$
\begin{aligned}
p_{\mathbf{T}}\left(I^{f}, \hat{G}^{f}, I^{r}, \hat{G}^{r}\right) & =\frac{h_{\mathbf{T}}\left(I^{f}, \hat{G}^{f}, I^{r}, \hat{G}^{r}\right)}{\sum_{I^{f}, \hat{G}^{f}, I^{r}, \hat{G}^{r}} h_{\mathbf{T}}\left(I^{f}, \hat{G}^{f}, I^{r}, \hat{G}^{r}\right)}, \\
p_{\mathbf{T}}\left(I^{f}, \hat{G}^{f}\right) & =\sum_{I^{r}, \hat{G}^{r}} p_{\mathbf{T}}\left(I^{f}, \hat{G}^{f}, I^{r}, \hat{G}^{r}\right), \\
p_{\mathbf{T}}\left(I^{r}, \hat{G}^{r}\right) & =\sum_{I^{f}, \hat{G}^{f}} p_{\mathbf{T}}\left(I^{f}, \hat{G}^{f}, I^{r}, \hat{G}^{r}\right) .
\end{aligned}
$$

\subsection{Multi-resolution Optimization}

In the proposed registration approach, the optimal transformation $\hat{\mathbf{T}}$ can be estimated by

$$
\hat{\mathbf{T}}=\arg \max _{\mathbf{T}} \mathrm{MI}(\mathbf{T}) .
$$

In order to accelerate the registration process and ensure the robustness of the proposed method, we exploit a multi-resolution approach based on the Gaussian Pyramid representation [11,2, 12. Rough estimates of $\hat{\mathbf{T}}$ can be found using downsampled images and treated as starting values for optimization at higher resolutions. Then the fine-tuning of the solution can be derived at the original image resolution. In this paper, the value of multi-dimensional mutual information at each resolution is maximized via the Powell's direction set method in multidimensions 13 .

\section{Implementation Details}

Signed-MDGM Map: In our implementation, the signed-MDGM map is computed by separating it into the positive and negative components. Then the two components are calculated by sequentially processing voxels in intensitydecreasing and intensity-increasing orders respectively.

During either procedure, we keep updating a Voronoi diagram and a (positive or negative) MDGM map. When a voxel $\mathbf{v}$ is processed, the Voronoi diagram is locally reconstructed by adding $\mathbf{v}$ into the Voronoi sites. We then update the MDGM map within the Voronoi cell $V(\mathbf{v})$ of $\mathbf{v}$. The reason for ignoring the exterior is illustrated as follows: Let $\mathbf{v}_{0}$ be a voxel in another Voronoi cell $V\left(\mathbf{v}^{\prime}\right)$ (i.e. $\left|\mathbf{v}-\mathbf{v}_{0}\right|>\left|\mathbf{v}^{\prime}-\mathbf{v}_{0}\right|$ ). Since $\mathbf{v}^{\prime}$ has been processed prior to $\mathbf{v}$, we 
have $I\left(\mathbf{v}^{\prime}\right) \geq I(\mathbf{v})$ (for decreasing order) or $I\left(\mathbf{v}^{\prime}\right) \leq I(\mathbf{v})$ (for increasing order). Therefore, we have $\left|\nabla_{d} I\left(\mathbf{v}^{\prime}, \mathbf{v}_{0}\right)\right|>\left|\nabla_{d} I\left(\mathbf{v}, \mathbf{v}_{0}\right)\right|$ and the MDGM value of $\mathbf{v}_{0}$ remains unchanged.

Finally, the positive and negative components of the signed-MDGM map are combined together according to their absolute values.

Multi-dimensional Mutual Information: For calculating multi-dimensional mutual information, the number of $4 \mathrm{D}$ joint histogram bins should be limited, due to the relatively high dimensionality. In practice, we have found that $4 \mathrm{D}$ histograms with 32 bins both for intensity and signed-MDGM dimensions performs good for registering two images of size $256 \times 256 \times 26$. (Note that the number of histogram bins may be tuned for downsampled images in multi-resolution registration process.)

\section{Experimental Results and Discussions}

To evaluate the multi-dimensional mutual information similarity measure on the novel two-element attribute vector space (hereafter referred to as $M I-4 D$ ) and the proposed multi-resolution registration method, we have performed three categories of experiments on different image modalities: MR-T1, MR-T2 and CT. Comparisons on capture range of the traditional mutual information similarity measure on intensity (hereafter referred to as MI-2D) [1,2] and MI-4D will be presented in Section 5.1. Section 5.2 will show the performance comparisons on registration robustness between the proposed method and the conventional MI-2D based method. The registration accuracy of the two methods will be demonstrated in Section 5.3 .

\subsection{Comparisons on Capture Range}

T1 - T2 (3D - 3D) Registration: Three pairs of clinical MR-T1 and MRT2 image volumes (datasets \#1, \#2 and \#3) were obtained from RREP. All these images have been rectified for intensity inhomogeneity and scaling, and hereafter they are referred to as T1-rec and T2-rec respectively. The size of these image volumes is $256 \times 256 \times 26$ voxels and the voxel size is around $1.26 \times 1.26 \times 4.1$ $\mathrm{mm}^{3}$. Note that all image pairs used in our experiments (T1-rec, T2-rec and CT) were first registered by the conventional multi-resolution MI based registration method and were then examined by an experienced consultant radiologist to ensure that the final alignments are correct and acceptable. This procedure was employed for a better presentation of the probing results and also for further facilitating the experiments that will be described in Section 5.2 .

Figures $2 \mathrm{a}$ and $2 \mathrm{~d}$ respectively plot the translational probes for registering the low resolution 4 (Level 2) testing image pairs from three datasets for MI-2D

\footnotetext{
${ }^{4}$ The definition of resolution levels in the Gaussian Pyramid representation follows the same line as in [11. The smoothing filter was $\{1,4,6,4,1\}$ in our experiments.
} 


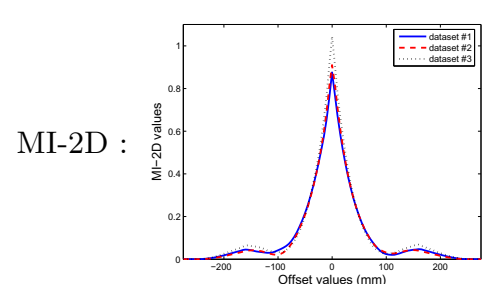

(a)

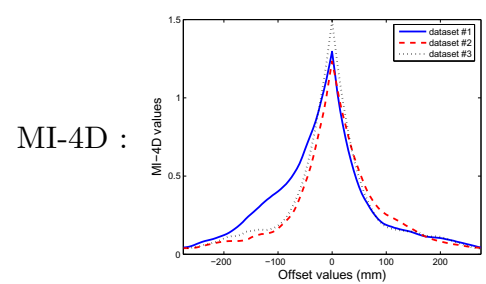

(d)

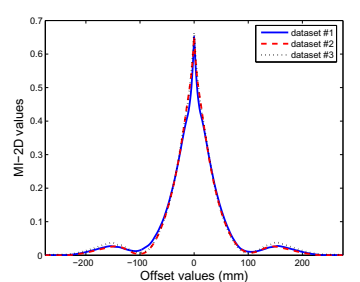

(b)

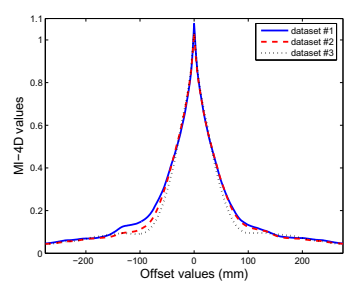

(e)

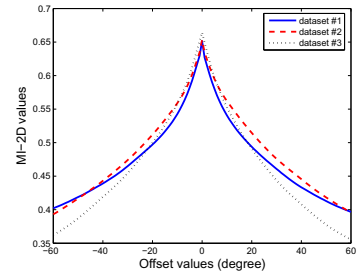

(c)

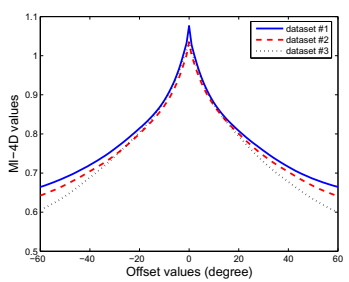

(f)

Fig. 2. Probing curves for $3 \mathrm{D}-3 \mathrm{D}$ registration on three T1-rec and T2-rec datasets (\#1, \#2 and \#3). Translational probes for registering the low resolution (Level 2) image pairs: (a) MI-2D and (d) MI-4D. Translational probes for registering the original resolution (Level 0) image pairs: (b) MI-2D and (e) MI-4D. Rotational probes for registering the original resolution (Level 0) image pairs: (c) MI-2D and (f) MI-4D

and MI-4D. At the original image resolution (Level 0), Figures $2 \mathrm{~b}$ and $2 \mathrm{p}$ plot the translational probes and Figures 2r and 2f plot the rotational probes based on MI-2D and MI-4D respectively. (Note that the number of histogram bins for MI-2D was set to $32 \times 32$ at all resolutions while that for MI-4D at Level 2 was set to $32 \times 32 \times 8 \times 8$, where 8 was for the signed-MDGM feature.)

As observed in Figures $2 \mathrm{a}$ and $2 \mathrm{~b}$, for the translational probes of MI-2D at different image resolutions, obvious local maxima occur when the misalignment of two images is relatively large. On the contrary, Figures $2 \mathrm{~d}$ and 2 e suggest that the shape of the probing curves based on MI-4D is improved and the capture ranges of MI-4D can be relative longer than those of MI-2D. This is because, with the proposed two-element attribute vector, regions with homogenous intensities (including the anatomical structures and background regions) can provide varying information related to the distance of a voxel to a certain object boundary. Therefore, when the misalignment increases, the MI-4D values would keep decreasing. With this finding, it is expected that the optimization procedure for registration will be benefited and the registration robustness can be increased. On the other hand, for the rotational probes, the capture ranges of MI-2D and MI-4D are comparable (see Figures 2rc and 2f).

CT - T1 (3D - 3D) Registration: Three pairs of clinical CT (around $512 \times 512 \times 30$ voxels and $0.65 \times 0.65 \times 4 \mathrm{~mm}^{3}$ ) and T1-rec image volumes (datasets \#1, \#2 and \#3) obtained from RREP were used for the experiments. The results 


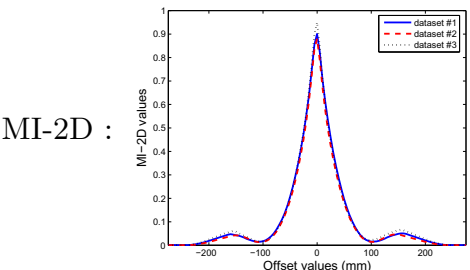

(a)

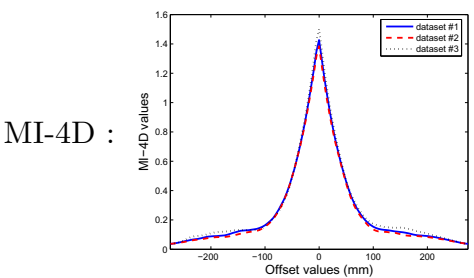

(d)

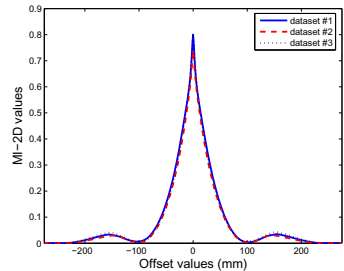

(b)

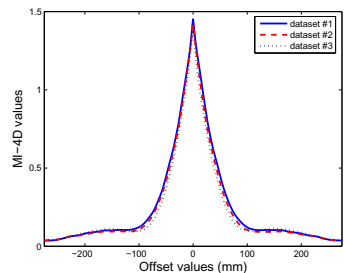

(e)

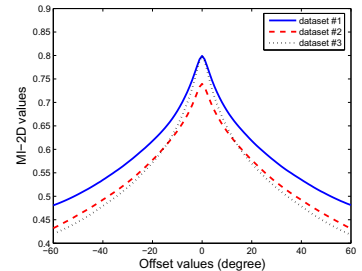

(c)

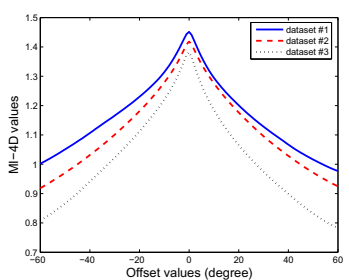

(f)

Fig. 3. Probing curves for 3D - 3D registration on three CT and T1-rec datasets (\#1, \#2 and \#3). Translational probes for registering the low resolution (Level 2) image pairs: (a) MI-2D and (d) MI-4D. Translational probes for registering the original resolution (Level 0) image pairs: (b) MI-2D and (e) MI-4D. Rotational probes for registering the original resolution (Level 0) image pairs: (c) MI-2D and (f) MI-4D

of translational probes are shown in Figures $3 \mathrm{3}$ (MI-2D) and $3 \mathrm{~d}$ (MI-4D) for the low resolution (Level 2) registration and in Figures $3 \mathrm{~b}$ (MI-2D) and 30 (MI-4D) for the original resolution (Level 0) registration. Figures $3 \mathrm{c}$ and 3 respectively plot the rotational probes based on MI-2D and MI-4D for the original resolution (Level 0). Similar results of the capture ranges are obtained as compared with $\mathrm{T} 1$ - T2 registrations.

\subsection{Performance Comparisons on Registration Robustness}

In order to study and compare the registration robustness of the proposed MI$4 \mathrm{D}$ based method and the conventional MI-2D based method, we have designed a series of randomized experiments for these two methods. The testing image pairs were the aforementioned three $\mathrm{T} 1$ - T2 datasets $(\# 1, \# 2$ and \#3) and three CT - T1 datasets (\#1, \#2 and \#3). The experiments took 100 tests on each testing image pair for either method. At each trial, the pre-obtained ground truth registration (see Section 5.1) of the testing image pair was perturbed by 6 uniformly distributed random offsets for all translational and rotational axes. The perturbed registration was then treated as the starting alignment. The random offsets for $\mathrm{X}$ and $\mathrm{Y}$ axes were drawn between $[-150,150] \mathrm{mm}$, while those for $\mathrm{Z}$ axis and each rotational axis were respectively drawn between $[-70,70] \mathrm{mm}$ and $[-0.35,0.35]$ radians (i.e. [-20, 20] degrees). (Note that for any testing dataset the same set of randomized starting alignments was used for both methods as a fair comparison.) 
Table 1. The success rates with the MI-2D based method and the MI-4D based method for all testing image pairs (T1 - T2 and CT - T1)

\begin{tabular}{|c|c|c|c|}
\hline & Testing & \multicolumn{2}{|c|}{ Success rate } \\
\cline { 2 - 4 } & dataset & MI-2D & MI-4D \\
\hline T1 & $\# 1$ & $68 \%$ & $89 \%$ \\
\cline { 2 - 4 } | & $\# 2$ & $65 \%$ & $95 \%$ \\
\cline { 2 - 4 } T2 & $\# 3$ & $66 \%$ & $81 \%$ \\
\hline CT & $\# 1$ & $66 \%$ & $94 \%$ \\
\cline { 2 - 4 } | & $\# 2$ & $63 \%$ & $88 \%$ \\
\cline { 2 - 4 } T1 & $\# 3$ & $70 \%$ & $94 \%$ \\
\hline
\end{tabular}

To evaluate each derived registration with respect to the ground truth registration, the translational error (which was the root-sum-square of the differences for three translational axes) and the rotational error (which was the real part of a quaternion) were computed. In our experiments, the threshold vector for assessing registration success was set to $\left(2 \mathrm{~mm}, 2^{\circ}\right)$, because registration errors below $2 \mathrm{~mm}$ and $2^{\circ}$ are generally acceptable by experienced clinicians [14, 15].

The success rates of the MI-2D based method and the MI-4D based method for all testing image pairs are listed in Table [1. It is suggested that the MI4D based method (Column MI-4D) consistently has higher success rates as compared with the MI-2D based method (Column MI-2D) for all testing image pairs. (Note that, due to the space limitation, we do not show the registration results of these 1200 randomized experiments in details.)

Based on these experiments, we also observed that the majority of failed cases for the MI-4D based method had about $180^{\circ}$ misalignment for one rotational axis, while registration errors for other axes were quite small. (It is meant that, after registration, the brain in the floating image was inverted along a rotational axis.) Oppositely, for the MI-2D based method, most of the failed cases had large translational and rotational misalignments simultaneously. This observation somehow implies that, along the translational axes, the capture ranges of MI-4D are longer than those of MI-2D.

\subsection{Registration Accuracy}

To precisely demonstrate the registration accuracy of the proposed registration method, similar randomized experiments described in Section 5.2 were performed on a pair of T1 and T2 image volumes obtained from the BrainWeb Simulated Brain Database [16] $\left(181 \times 217 \times 181\right.$ voxels, $1 \times 1 \times 1 \mathrm{~mm}^{3}$ and the noise level was $3 \%)$. Note that this image pair is perfectly aligned. The experiments took 50 tests for the MI-4D based method, as well as for the MI-2D based method as a comparison. For perturbation, the random offsets for each translational axis were drawn between $[-30,30] \mathrm{mm}$, and those for each rotational axis were drawn 
Table 2. The means and standard deviations of the registration accuracies of the MI$2 \mathrm{D}$ based method and the MI-4D based method for a BrainWeb T1 - T2 image pair with $3 \%$ noise level

\begin{tabular}{|c|ccc|ccc|}
\hline \multirow{2}{*}{ Method } & \multicolumn{3}{|c|}{ Translation $\left(10^{-3} \mathrm{~mm}\right)$} & \multicolumn{3}{c|}{ Rotation $\left(10^{-3}\right.$ degrees $)$} \\
\cline { 2 - 7 } & $\Delta t_{x}$ & $\Delta t_{y}$ & $\Delta t_{z}$ & $\Delta \theta_{x}$ & $\Delta \theta_{y}$ & $\Delta \theta_{z}$ \\
\hline MI-2D & $-0.40 \pm 0.71$ & $-0.62 \pm 1.41$ & $4.15 \pm 1.88$ & $0.63 \pm 1.85$ & $0.47 \pm 1.78$ & $0.04 \pm 1.55$ \\
\hline MI-4D & $-1.09 \pm 0.60$ & $-1.10 \pm 0.79$ & $4.14 \pm 2.05$ & $1.02 \pm 1.72$ & $0.85 \pm 1.61$ & $0.02 \pm 1.82$ \\
\hline
\end{tabular}

between $[-0.17,0.17]$ radians (i.e. $[-10,10]$ degrees). It should be noticed that all registrations obtained by either method are successful.

The means and standard deviations of the registration accuracies for each transformation parameter for these 100 experiments are lists of in Table 2, where Row MI-2D is for the MI-2D based method and Row MI-4D is for the MI$4 \mathrm{D}$ based method. According to the table, the accuracies of the MI-2D based method and the MI-4D based method are comparable and acceptably high. Both methods can achieve sub-voxel level registration accuracy.

\section{Conclusion}

To conclude, this paper has designed a new spatial feature, namely maximum distance-gradient-magnitude (MDGM), for registration tasks. The MDGM feature encodes spatial information for each voxel in an image at a global level. Then, we have improved the conventional mutual information (MI)-based registration framework by integrating the MDGM feature with intensity and setting a two-element attribute vector to each voxel in individual images. Multidimensional mutual information has been adopted as a similarity measure to the attribute vector space. To increase computation efficiency and robustness of the proposed method, the registration procedure has been a multi-resolution iterative process.

The experimental results on clinical 3D MR-T1, MR-T2 and CT datasets have indicated that the proposed method has relatively longer capture ranges than the conventional MI-based method at different image resolutions. Moreover, a large number of (around 1200) randomized experiments on precisely registered clinical image pairs have demonstrated that the success rates of our method are consistently higher than those of the traditional MI-based method. It has been also shown that the registration accuracy of the new method is acceptably high and obtains sub-voxel level.

\section{Acknowledgements}

The authors would like to acknowledge the support from the Hong Kong Research Grants Council (HK RGC) under grant (HKUST6155/03E). 


\section{References}

1. Maes, F., Collignon, A., et al.: Multimodality Image Registration by Maximization of Mutual Information. IEEE Trans. Med. Img. 16 (1997) 187-198

2. Wells, W., Viola, P., et al.: Multi-Modal Volume Registration by Maximization of Mutual Information. Medical Image Analysis 1 (1996) 35-51

3. Penney, G., Weese, J., et al.: A Comparison of Similarity Measures for Use in 2D-3D Medical Image Registration. IEEE Trans. Med. Img. 17 (1998) 586-595

4. Pluim, J., Maintz, J., Viergever, M.: Image Registration by Maximization of Combined Mutual Information and Gradient Information. IEEE Trans. Med. Img. 19 (2000) 809-814

5. Pluim, J., Maintz, J., Viergever, M.: Mutual-Information-Based Registration of Medical Images: A Survey. IEEE Trans. Med. Img. 22 (2003) 986-1004

6. Rueckert, D., Clarkson, M., et al.: Non-rigid registration using higher-order mutual information. In: Proc. SPIE, Medical Imaging: Image Processing. Volume 3979. (2000) 438-447

7. Butz, T., Thiran, J.P.: Affine registration with feature space mutual information. In: MICCAI. (2001) 549-556

8. Borgefors, G.: Distance transformations in arbitrary dimensions. Comput. Vision, Graphics, and Image Processing 27 (1984) 321-345

9. Maes, F., Vandermeulen, D., Suetens, P.: Medical Image Registration Using Mutual Information. Proceedings of the IEEE 91 (2003) 1699-1722

10. Bishop, C.: Neural Networks for Pattern Recognition. Oxford U. Press (1995)

11. Burt, P., Adelson, E.: The Laplacian Pyramid as a Compact Image Code. IEEE Trans. Comms. 31 (1983) 532-540

12. Cole-Rhodes, A., Johnson, K., et al.: Multiresolution Registration of Remote Sensing Imagery by Optimization of Mutual Information Using a Stochastic Gradient. IEEE Trans. Image Processing 12 (2003) 1495-1511

13. Press, W., Teukolsky, S., et al.: Numerical Recipes in C, 2nd Edition. Cambridge University Press (1992)

14. Hajnal, J.V., Hill, D.L.G., Hawkes, D.J.: Medical Image Registration. CRC Press LLC (2001)

15. Zhu, Y., Cochoff, S.: Likelihood Maximization Approach to Image Registration. IEEE Trans. Image Processing 11 (2002) 1417-1426

16. Collins, D., Zijdenbos, A., et al.: Design and Construction of a Realistic Digital Brain Phantom. IEEE Trans. Med. Img. 17 (1998) 463-468 